\title{
A Survey for Low-Surface-Brightness Galaxies Around M31. I. The Newly Discovered Dwarf Andromeda V
}

\author{
Taft E. Armandroff \\ Kitt Peak National Observatory, National Optical Astronomy Observatories ${ }^{1}$, \\ P.O. Box 26732, Tucson, AZ 85726 \\ James E. Davies \\ Kitt Peak National Observatory, National Optical Astronomy Observatories ${ }^{1}$, \\ P.O. Box 26732, Tucson, AZ 85726; \\ and \\ Department of Astronomy, University of Wisconsin, Madison, WI 53706 \\ and \\ George H. Jacoby ${ }^{2}$ \\ Kitt Peak National Observatory, National Optical Astronomy Observatories ${ }^{1}$, \\ P.O. Box 26732, Tucson, AZ 85726
}

Received

accepted

Accepted by The Astronomical Journal, November 1998 issue

\footnotetext{
${ }^{1}$ The National Optical Astronomy Observatories are operated by AURA, Inc., under cooperative agreement with the National Science Foundation.

${ }^{2}$ Visiting Astronomer, Steward Observatory, University of Arizona
} 


\begin{abstract}
We present images and a color-magnitude diagram for And V, a new dwarf spheroidal companion to M31 that was found using a digital filtering technique applied to $1550 \mathrm{deg}^{2}$ of the second Palomar Sky Survey. And V resolves into stars easily in follow-up 4-m $V$ - and $I$-band images, from which we deduce a distance of $810 \pm 45 \mathrm{kpc}$ using the tip of the red giant branch method. Within the uncertainties, this distance is identical to the Population II distances for M31 and, combined with a projected separation of $112 \mathrm{kpc}$, provides strong support for a physical association between the two galaxies. There is no emission from And V detected in $\mathrm{H} \alpha, 1.4 \mathrm{GHz}$ radio continuum, or IRAS bandpasses, and there is no young population seen in the color-magnitude diagram that might suggest that And $\mathrm{V}$ is an irregular. Thus, the classification as a new dwarf spheroidal member of the Local Group seems secure. With an extinction-corrected central surface brightness of $25.2 \mathrm{~V} \mathrm{mag} / \mathrm{arcsec}^{2}$, a mean metal abundance of $[\mathrm{Fe} / \mathrm{H}] \approx$ -1.5, and no evidence for upper AGB stars, And V resembles And I \& III.
\end{abstract}

Subject headings: galaxies: dwarf — galaxies: individual (And V, M31) — galaxies: stellar content — galaxies: structure — Local Group — surveys — techniques: image processing 


\section{Introduction}

A complete and accurate knowledge of dwarf galaxies in the Local Group is necessary for many problems in galaxy formation and cosmology (see Ferguson \& Binggeli 1994, Gallagher \& Wyse 1994). For example, the faint end of the luminosity function in large clusters of galaxies is more populated than that of the Local Group (Trentham 1998). Because this result may be explained by incompleteness and/or small number statistics in the Local Group, it remains unknown whether low-luminosity galaxies form more frequently in denser environments or not. As another example, van den Bergh (1994) found a correlation between the Galactic dwarfs' galactocentric distances and the prominence of an intermediate-age population. This may reflect the effects of ram pressure stripping or photoionization via the early Milky Way's integrated UV flux on the inner dwarf spheroidals. However, our ability to confirm or rule out this trend in the independent case of M31 is hampered by its small number of known companions.

The Milky Way has nine low-surface-brightness gas-poor companions; these are usually called dwarf spheroidal galaxies. Dwarf spheroidals are now considered low-luminosity dwarf elliptical galaxies. The empirical correlations followed by dwarf spheroidals join smoothly onto the dwarf elliptical correlations (e.g., central surface brightness vs. absolute magnitude, mean metal abundance vs. absolute magnitude). M31 has three dwarf spheroidal companions (And I, II \& III) and three dwarf elliptical companions (NGC 147, 185 \& 205) $)^{\beta}$. This relatively small number would seem to suggest a deficit of dwarf spheroidal companions of M31 compared to the Milky Way. Is this a real effect or simply due to the fact that the Milky Way has been searched more thoroughly than M31? Alternatively, it is possible that M31's environment was more conducive to producing more luminous dwarfs

\footnotetext{
${ }^{3}$ See Gallagher \& Wyse (1994), Armandroff (1994), and Da Costa (1998) for recent reviews on the dwarf companions to the Milky Way and M31.
} 
like NGC 147, 185 and 205, rather than very low luminosity dwarfs. Another possibility is that M31 was more effective than the Milky Way in disrupting and accreting its original population of low-luminosity dwarfs.

The current census of M31 dwarf spheroidals resulted from the pioneering survey of van den Bergh (1972a, 1974). He visually searched 700 square degrees around M31 using IIIaJ photographic plates taken with the Palomar 48-inch Schmidt telescope. Three dwarf spheroidal candidates were found (And I, II \& III). One smaller, higher surface brightness object was also found (And IV); subsequent work confirmed van den Bergh's (1972a) conclusion that it is not a dwarf spheroidal (Jones 1993). And I, II \& III were found to resolve into stars at approximately the same magnitude as the M31 companion NGC 185 (van den Bergh 1972b, 1974), supporting the notion that these three galaxies are physically associated with M31. As more precise distances to And I, II \& III have become available (e.g., Armandroff et al. 1993, Da Costa et al. 1996), the conclusion that these galaxies are located in the outer reaches of M31's halo has remained valid.

For the Galactic dwarf spheroidals, all three components of their galactocentric distance are known. However, only projected distances from the center of M31 are known for And II \& III (see Da Costa et al. 1996 for a precise line-of-sight distance for And I, based on the magnitude of the horizontal branch, and the resulting limits on its true three-dimensional distance from the center of M31). Figure 6 of Armandroff (1994) compares the projected galactocentric distance distribution of the M31 dwarf spheroidals with that of their Galactic counterparts. Van den Bergh's (1972a, 1974) survey begins to lack complete areal coverage at a projected galactocentric distance of $\sim 110 \mathrm{kpc}$, and it has no coverage beyond a projected galactocentric distance of $\sim 260 \mathrm{kpc}$. Since Leo I and II are located $>200 \mathrm{kpc}$ from the Galactic center, some M31 dwarfs with very large galactocentric distances may be outside van den Bergh's survey area. Finally, because And III with a central surface 
brightness of $25.3 \mathrm{~V} \mathrm{mag} / \operatorname{arcsec}^{2}$ was found by van den Bergh, we know that his survey was sensitive to quite low surface brightness. However, the central surface brightness to which van den Bergh's (1972a, 1974) survey is complete is not known.

In order to find possible additional M31 low-surface-brightness companions, we undertook a new search of the area around M31. Our search uses the Second Palomar Sky Survey (POSS-II; Reid et al. 1991, Reid \& Djorgovski 1993), which has higher resolution and extends substantially deeper than its predecessor. The availability of the POSS-II in digital form (Lasker \& Postman 1993) allows the use of digital image processing techniques. These techniques may enable the detection of dwarf galaxies of even lower central surface brightness than van den Bergh's (1972a, 1974) completeness limit. The digital POSS-II also facilitates the search of an arbitrarily large area of sky around M31.

This paper will provide a brief description of our search strategy and techniques. This will be followed by the first results of our survey, in which we identify a new dwarf galaxy associated with M31.

\section{Search Methodology}

The POSS-II data used for the survey consists of red IIIaF + RG610 plates that were digitized at STScI. The red bandpass was chosen for searching for dSph galaxies because most of their light is typically emitted by red giants and red horizontal branch stars. The fainter sky and broader bandpass of the blue plates suggest that these might have similar detection efficiency, but at the time of our study only the red plates were accessible digitally. The plates are 6.6 degrees on a side with 6.3 degrees of useable emulsion. They are spaced 5 degrees between plate centers which gives a 1.3 degree overlap area for cross-checking candidates to eliminate spurious detections. Having this relatively large overlap is useful 
considering the optical vignetting effects in the outer regions of the plates as discussed by Reid et al. (1991). Each digitized plate is subdivided into a grid of $7 \times 71 \mathrm{deg}^{2}$ images (each 3564 pixels on a side) to make the image processing more manageable. The 49 images per plate consequently have overlaps of 7 arcmin which is larger than the FWHM of a typical dwarf galaxy profile at the distance of M31; therefore no objects are missed due to being close to an edge.

Each $1 \operatorname{deg}^{2}$ image is filtered separately in a four-step process for the purpose of increasing the signal-to-noise of low-surface-brightness (LSB) features. The procedure was optimized for the detection of And II \& III. These steps are:

1. A fourth order polynomial is fit to and subtracted from the background of each image in order to make the background as flat as possible. It should be noted that these plates are remarkably flat on scales of several $\mathrm{cm}$, corresponding to about a degree. The background needs to be as flat as possible due to the way that high-surface-brightness (HSB) objects are removed in step 2. It was found through successive trials of fitting polynomials of different order that a fourth order polynomial flattened the plate well enough without running the risk of removing LSB features on the scale of a few arcmin. Values $2 \sigma$ higher than the image median are not used in the fitting so that the polynomial fit is dominated by the background and not by stars and galaxies in the image (where $\sigma$ is the standard deviation).

2. Stars on the images are removed by a clipping operation that calculates the median value of the full image and replaces all values $>0.75 \sigma$ above and $<0.75 \sigma$ below the median with the median value. The positive clip has the effect of removing all HSB objects - stars, obvious galaxies and clusters of galaxies - that hamper the detection process. The negative clip gets rid of darkened rings caused by dust and other plate defects. The clipping operation works well since it has no preference for the size or 
shape of the HSB object - it removes everything. The value of $0.75 \sigma$ was found to be optimal in that it removes much of the "wings" of large, bright stars but does not clip too low and run the risk of diminishing the peaks of our test dSph galaxies, And II \& III.

3. The images are then filtered with a square spatial median filter of size $77 \times 77$ arcsec that moves across the image replacing the central pixel value with the median of the $77 \times 77$ pixel values contained in the box. Davies et al. (1994) found that in order to optimally enhance and detect an LSB disk galaxy, their cross-correlation filter size and the scale length of the galaxy ought to be roughly equal within a factor of 2 . We confirm this for a median filter and LSB dwarf galaxies and find that for dSph with exponential scale lengths in the 45 to 94 arcsec range defined by the known Andromeda dwarfs (Caldwell et al. 1992), an optimal median filter size is $77 \times 77$ arcsec.

4. Detection of LSB dwarf galaxies is carried out by visually comparing the filtered images with the raw images and selecting candidates that resemble And II \& III on the raw and processed images. LSB features are prominent on the processed images but are extremely weak, and in many cases invisible, on the unprocessed images. And II \& III are trivial to detect on processed POSS-II images (processed POSS-I images easily reveal all three M31 dSph). Images of And II \& And III from the POSS-II, raw and after processing, are shown in Figure 1 (And I is not shown because the relevant digitized POSS-II image is not yet available).

EDITOR: PLACE FIGURE 1 HERE.

The survey area was selected based on the area searched by van den Bergh (1974) and was extended out 6-10 degrees farther in all directions for the completeness reasons stated 
in the Introduction. Figure 2 shows the area that has been searched so far as shaded boxes, with the van den Bergh area outlined. The area searched consists of 51 plates that cover approximately $1550 \mathrm{deg}^{2}$.

\section{EDITOR: PLACE FIGURE 2 HERE.}

Since the image processing enhances any density feature on the plate that is the proper size and low enough surface brightness (a few percent above sky), and because the survey area is very large, identifying and eliminating contaminants that masquerade as LSB dwarfs is a daunting task. The biggest offenders are ghosts produced from the reflection of bright stars in the telescope optics and Galactic reflection nebulae (also referred to as Galactic cirrus clouds; see Bremmes, Binggeli \& Prugniel 1998). In many cases follow-up observations are the only way to eliminate candidates. Fortunately, many detections are asymmetric and lack a smooth exponential profile, rendering them inconsistent with an LSB dwarf galaxy signature. Therefore, such objects can be eliminated from the candidate list. The remainder, however, require follow-up CCD imaging, which is described in the next section.

\section{Follow-Up Observations}

The remainder of this paper will concentrate on one excellent candidate that was found at the following celestial coordinates: $\alpha_{2000}=1: 10: 17.1, \delta_{2000}=+47: 37: 41$. These correspond to Galactic coordinates of: $l=126.2^{\circ}, b=-15.1^{\circ}$. There are no previously catalogued galaxies/objects at these coordinates according to the NASA/IPAC Extragalactic Database

\footnotetext{
${ }^{4}$ The NASA/IPAC Extragalactic Database (NED) is operated by the Jet Propulsion Laboratory, California Institute of Technology, under contract with the National Aeronautics
} 
and the SIMBAD database. Because other "newly found" nearby dwarf galaxies have occasionally turned out to be rediscoveries, we have inspected a number of lists that may contain such galaxies in order to verify that this object is indeed new, including Karachentseva \& Karachentsev (1998), Nilson (1973), Schmidt \& Boller (1992), Ellis et al. (1984), Weinberger (1995), and Schombert et al. (1992). Following van den Bergh (1972a), we will call this new system Andromeda V. Figure 2 shows where And V falls in the search area.

Figure 3 shows And V on the digitized POSS-II, raw and after processing. One sees a strong resemblance between And V and the known M31 dwarf spheroidals And II \& III on the raw and processed POSS-II images (see Figure 1). We examined the POSS-I prints and found that And V is not visible on them; And I, II \& III are not clearly visible on the POSS-I prints either. And I \& II are easily visible on the POSS-II transparencies. And III, which is of lower central surface brightness than And I \& II (Caldwell et al. 1992), is just visible on the POSS-II. And V appears to be of lower surface brightness still and is marginally discernible on the POSS-II provided that the coordinates are known a priori. Karachentseva \& Karachentsev (1998) also noted that And I, II \& III are visible on the POSS-II.

\section{EDITOR: PLACE FIGURE 3 HERE.}

The first step in clarifying the nature of a candidate from the sky survey is smalltelescope CCD imaging. Such imaging eliminates candidates that are really ghosts from bright stars on the POSS-II. Other potential "contaminants" that can be eliminated via small-telescope imaging include: distant clusters of galaxies, planetary nebulae, and distant

and Space Administration. 
low-surface-brightness spirals.f And V was imaged at the KPNO 0.9-m telescope with the CCD Mosaic Imager (see Armandroff et al. 1998) in the $R$ band for 600 seconds on September 28, 1997. The scale was 0.43 arcsec/pixel, and the seeing was 1.4 arcsec FWHM. In the 0.9-m data, And V looks like a low-surface-brightness galaxy and shows incipient resolution into stars.

And $\mathrm{V}$ was imaged at the KPNO 4-m telescope prime focus with the T2KB CCD on November 2, 1997 (see Massey et al. 1997 for a description of the imager). The scale was $0.42 \mathrm{arcsec} / \mathrm{pixel}$, and the seeing was 1.0 arcsec FWHM. Three $V$ exposures of 900 sec each and seven $I$ exposures of $300 \mathrm{sec}$ each were obtained. It was photometric and $\sim 15$ Landolt (1992) standards in three fields were observed. The combined 4-m $V$ image is displayed in Figure 4 (the central $1000 \times 1000$ pixels). And V resolves nicely into stars on both the $V$ and the $I$ images. One sees a smooth stellar distribution. In these images, And V resembles the other M31 dwarf spheroidals (see Figure 1 of Mould \& Kristian 1990 and/or Figure 1 of Armandroff et al. 1993). And V does not look lumpy or show obvious regions of star formation that would suggest a dwarf irregular classification. No obvious globular clusters are seen in And V, though none are expected for a low-luminosity dwarf spheroidal (e.g., Sec. 4.3 of Da Costa \& Armandroff 1995).

\section{EDITOR: PLACE FIGURE 4 HERE.}

In order to look for possible ionized gas, And V was observed with the KPNO 4-m telescope in $\mathrm{H} \alpha$ narrow-band and $R$ on October 21, 1997 with the CCD Mosaic Imager. The H $\alpha$ filter used has a central wavelength of $6569 \AA$ and a width of $80 \AA$ FWHM in the $\mathrm{f} / 3.2$ beam of the telescope. Five narrow-band exposures of $600 \mathrm{sec}$ each and five

\footnotetext{
${ }^{5}$ Note that we have not completed follow-up imaging of all of our candidate LSB dwarfs. We will report follow-up imaging of other candidates in a future paper.
} 
$R$ exposures of $400 \mathrm{sec}$ each were obtained. The average seeing was 1.2 arcsec FWHM, sampled at 0.26 arcsec/pixel. We computed scaling ratios between the combined $\mathrm{H} \alpha$ and $R$ images and performed the subtraction. Figure 5 shows both the $\mathrm{H} \alpha$ image and the continuum-subtracted $\mathrm{H} \alpha$ image. No diffuse $\mathrm{H} \alpha$ emission or $\mathrm{H}$ II regions were detected in And V. Some residuals are present in this image due to the somewhat different behavior of the PSF in $R$ and $\mathrm{H} \alpha$. There are a few small positive residuals, but these are all detected strongly in $V, R$, and $I$ and therefore are not $\mathrm{H}$ II regions or planetary nebulae. Their slightly extended nature suggests that they are distant galaxies. The lack of $\mathrm{H} \alpha$ emission in And V likely rules out significant current star formation, reinforcing our conclusion, based on And V's appearance on the broad-band images, that it is a dwarf spheroidal galaxy rather than a dwarf irregular. Almost all Local Group dwarf irregulars are easily detected in $\mathrm{H} \alpha$ (e.g., Kennicutt 1994).

\section{EDITOR: PLACE FIGURE 5 HERE.}

We also examined the IRAS maps of the And $\mathrm{V}$ region, using the FRESCO data product from IPAC . And V is not detected in any of the IRAS far-infrared bands. And I, II \& III are not detected by IRAS either. Far-infrared emission, as seen by IRAS, is the signature of warm dust. Some Local Group dwarf irregular galaxies, such as NGC 6822, IC 1613 and WLM, are detected by IRAS (Rice et al. 1988). Hence the non-detection of And $\mathrm{V}$ by IRAS is further evidence that it does not contain substantial amounts of dust and gas. However, many of the less active, less luminous Local Group dwarf irregulars are not detected by IRAS either. Consequently, the lack of far-infrared emission from And V is probably a weaker constraint on the presence of an interstellar medium than the lack of $\mathrm{H} \alpha$ emission.

\footnotetext{
${ }^{6}$ IPAC is funded by NASA as part of the IRAS extended mission under contract to JPL.
} 
And $\mathrm{V}$ is not detected in the NRAO VLA $1.4 \mathrm{GHz}$ radio continuum survey (Condon et al. 1998). This is as expected because none of the Local Group dwarf spheroidals are detected in this survey, and even most Local Group dwarf irregulars are not detected. IC 10 is easily detected in the $1.4 \mathrm{GHz}$ survey, but it has an exceptional star-formation rate and disturbed interstellar medium (Yang \& Skillman 1993, Massey \& Armandroff 1995).

We know nothing about the $\mathrm{H}$ I content of And V via $21 \mathrm{~cm}$ observations. Either an $\mathrm{H}$ I detection or a strict upper limit would be valuable.

\section{Color-Magnitude Diagram}

The next step in ascertaining the nature of And $\mathrm{V}$ is the construction of a colormagnitude diagram for its member stars in order to reveal its distance and stellar populations characteristics. Instrumental magnitudes were measured on the And V 4-m images using the IRAF implementation of the DAOPHOT crowded-field photometry

program (Stetson 1987, Stetson et al. 1990). The standard DAOPHOT procedure was used, culminating in multiple iterations of ALLSTAR. Because substantial point-spread-function variations occur between the middle and corners of the CCD frames, only the central $1000 \times 1000$ region was analyzed. Stars with anomalously large values of the DAOPHOT parameter CHI were removed from the photometry lists.

Large-aperture photometry was performed for the $\sim 15$ well-exposed Landolt (1992) photometric standard stars. Assuming mean atmospheric extinction coefficients for Kitt Peak, photometric transformation equations were derived. Besides the zeropoint, only a linear color term of small size was needed in each filter. The And V ALLSTAR magnitudes were placed on the standard system via large-aperture photometry of several bright, uncrowded stars on the And V images in each filter. 
Initial color-magnitude diagrams for the parts of the images dominated by And V stars revealed a red giant branch, which is absent in the outer regions of the images. We used the spatial distribution of the stars in the red-giant region of the color-magnitude diagram to determine a reasonably precise center for And V (see Figure 4)).

The color-magnitude diagram of And $\mathrm{V}$ is illustrated in Figure 6. The left panel shows the color-magnitude diagram for the region where And V stars dominate over field contamination, a circle of radius 170 pixels or 71 arcsec centered on And V (chosen as a compromise between maximizing the number of And V stars and minimizing field contamination; hereafter referred to as the And V region). The upper red giant branch of And $\mathrm{V}$ is visible in this left panel. The right panel displays the color-magnitude diagram for the portion of the frames where the And V contribution is negligible, that area outside a radius of 400 pixels or 168 arcsec from the center of And V (hereafter referred to as the field region). The And $\mathrm{V}$ giant branch is absent from the right panel. Instead, a broad swath of field stars covers most of the color-magnitude plane. Considering that the field region represents 5.5 times more area than the And $\mathrm{V}$ region, most of the stars that deviate from red giant branch in the left panel can be interpreted as field contamination. As a rough comparison, the colors and magnitudes of the And V stars in the left panel of Figure 6 are similar to those of the And III bright red giants (see Figure 5 of Armandroff et al. 1993). This similarity, which we will investigate carefully below, substantially strengthens the evidence that And V is indeed associated with M31.

\footnotetext{
${ }^{7}$ The $\mathrm{x}, \mathrm{y}$ coordinates of this center were transformed to celestial coordinates using the HST Guide Star Catalog. This is the origin of the celestial coordinates for And V given in the first paragraph of Sec. 3. The uncertainty in the coordinates is dominated by the error in determining the centroid of likely member giants and is roughly 5 arcsec.
} 


\section{EDITOR: PLACE FIGURE 6 HERE.}

A distance can be derived for And $\mathrm{V}$ based on the $I$ magnitude of the tip of the red giant branch (Da Costa \& Armandroff 1990, Lee et al. 1993). Figure 0 l shows I luminosity functions for the And V region and for the field region (the same regions as used in constructing the color-magnitude diagrams in Figure (6). Based on the magnitude at which the And V luminosity function begins to rise strongly above that of the field, the $I$ magnitude of the red giant branch tip is $20.85 \pm 0.10$; this value is supported by the apparent location of the tip in the color-magnitude diagram (Figures 6 and 8 ). For metal-poor systems such as And V (see below), the red giant branch tip occurs at $M_{I}=$ -4.0 (Da Costa \& Armandroff 1990, Lee et al. 1993). We adopt an interstellar reddening for And $\mathrm{V}$ of $\mathrm{E}(B-V)=0.16 \pm 0.03$ from the Burstein \& Heiles (1982) extinction maps. Assuming $A_{V}=3.2 \times \mathrm{E}(B-V)$ and the $\mathrm{E}(B-V)$ to $\mathrm{E}(V-I)$ conversion of Dean, Warren \& Cousins (1978), $\mathrm{E}(V-I)=0.21$ and $A_{I}=0.30$. The above values then yield a true distance modulus for And $\mathrm{V}$ of $24.55 \pm 0.12$ and a distance of $810 \pm 45 \mathrm{kpc}$. These error estimates do not include any systematic uncertainty in the zeropoint of the adopted distance scale.

\section{EDITOR: PLACE FIGURE 7 HERE.}

How does this And V distance compare with that of M31? The most directly comparable distance determinations for M31 are based on either red giant branch tip stars or RR Lyraes in the M31 halo or horizontal branch stars in M31 globular clusters. Such distance determinations are discussed in Da Costa et al. (1996) in order to compare the distances of M31 and And I. On the same distance scale as used here, the red giant branch tip stars (Mould \& Kristian 1986) and RR Lyraes (Pritchet \& van den Bergh 1988) agree well and yield an M31 distance of $760 \pm 45 \mathrm{kpc}$. On the other hand, the horizontal branch magnitudes for eight globular clusters observed by HST (Fusi Pecci et al. 1996) suggest 
an M31 distance of $850 \pm 20 \mathrm{kpc}$ on the same scale. The Cepheid distance to M31 is 770 kpc (Freedman \& Madore 1990); Lee et al. (1993) and Sakai et al. (1997) have shown that the Cepheid distance scale and the tip of the red giant branch scale are consistent. Our distance for And $\mathrm{V}$ of $810 \pm 45 \mathrm{kpc}$ implies that And V is located at the same distance along the line of sight as M31 to within the uncertainties. And V's projected distance from the center of M31 is $112 \mathrm{kpc}$; And I, II \& III have projected M31-centric distances of 46, 144 and $69 \mathrm{kpc}$, respectively. The above line-of-sight and projected distances strongly suggest that And V is indeed associated with M31.

In order to investigate the stellar populations in And $\mathrm{V}$, we have overplotted its color-magnitude diagram with fiducials representing the red giant branches of Galactic globular clusters that span a range of metal abundance (Da Costa \& Armandroff 1990). For an old stellar population (as demonstrated below for And V), the color of the red giant branch is primarily determined by metal abundance. Figure 8 shows $I, V-I$ color-magnitude diagrams for the And V and field regions, with fiducials for M15 ([Fe/H] $=-2.17)$, M2 $(-1.58)$, NGC 1851 (-1.16), and 47 Tuc (-0.71, dotted line). Most of the And V giants lie near or slightly redwards of the M2 fiducial. Thus, the mean metal abundance of And V is approximately -1.5 . This metallicity is normal for a dwarf spheroidal (e.g., see Figure 9 of Armandroff et al. 1993).

\section{EDITOR: PLACE FIGURE 8 HERE.}

In Figure 8, the region of the color-magnitude diagram substantially blueward of the lowest metallicity fiducial (M15) is empty. Since the $V$ data reach significantly deeper than the $I$ data, stars in this region of the color-magnitude diagram should be easily detected. Thus, no bright blue stars appear to be present in And V. Using the Bertelli et al. (1994) isochrones, we conservatively estimate that no stars younger than at least $200 \mathrm{Myr}$ are present in the And V region of Figure 8. Local Group dwarf irregular galaxies contain 
many stars younger than 200 Myr. As a conservative example, LGS 3, which is considered a transition object with both dwarf spheroidal and dwarf irregular characteristics, has stars of age 100 Myr (Mould 1997). Thus, the lack of young stars is strong evidence that And V is a dwarf spheroidal and not a dwarf irregular. We hope to obtain deeper imaging of And $\mathrm{V}$, particularly in the $B$ band, in order to place stronger limits on the youngest stars in And V.

Using the luminosities and numbers of upper asymptotic giant branch (AGB) stars in a metal-poor stellar system, one can infer the age and strength of its intermediate-age component (Renzini \& Buzzoni 1986). Armandroff et al. (1993) have analyzed the luminosity functions of And I \& III and deduced that the fraction of each galaxy's luminosity contributed by an intermediate-age population is $10 \pm 10$ percent (where intermediate age denotes 3-10 Gyr old on an age scale where the Galactic globular clusters are $\approx 14$ Gyr old). Because And II contains upper AGB carbon stars (Aaronson et al. 1985), its intermediate-age component seems more substantial (though a quantitative analysis has not been performed). Intermediate-age components of varying prominence are present in the Galactic dwarf spheroidals, ranging from $\sim 75 \%$ of all stars in Carina to $\sim 0 \%$ in Ursa Minor (e.g., Da Costa 1998). Armandroff et al. (1993) used upper AGB stars with $M_{\text {bol }}$ values between -3.8 and -4.6 to constrain the number of stars with ages between 3 and 10 Gyr. Observations of And I revealed $4 \pm 4$ such stars; And III contains $3 \pm 2.9$ such stars. For And $\mathrm{V}$, the above $M_{\mathrm{bol}}$ limits correspond to $19.75 \leq I<20.45$. Examination of the And V luminosity function (Figure 7) indicates no significant excess of And V stars over the field in this magnitude interval. After appropriate color limits and field subtraction, there are $0.9 \pm 3.0$ excess stars in the And V region within the upper AGB magnitude limits. Thus, And V does not contain significant numbers of upper AGB stars. Therefore, it does not have a prominent intermediate-age population; in this sense, it is similar to And I \& III. 
Because dwarf spheroidal galaxies are faint compared to the terrestrial night sky, investigation of their surface brightness profiles requires very careful attention to flat fielding and background subtraction (see Sec. 3.1 of Caldwell et al. 1992). The current data are not well suited to determination of And V's surface brightness profile. However, it is possible to derive And V's central surface brightness via large-aperture photometry in the core of And $\mathrm{V}$. As a first step, we subtracted all stars brighter than $V=22$ from the And $\mathrm{V} V$ image, since they are field contamination. We then performed digital aperture photometry with an aperture radius of 20 arcsec and a sky annulus near the edge of the image (193-204 arcsec). This yielded an And V apparent $V$ central surface brightness of $25.7 \mathrm{mag} / \mathrm{arcsec}^{2}$. This result is insensitive to the precise specification of the sky annulus, and changing the radius of the aperture by 5 arcsec results in surface brightness changes at the 0.1 mag level. And V has a fainter apparent central surface brightness than And I, II \& III (24.9, 24.8, and 25.3 $V$ mag/arcsec ${ }^{2}$, respectively; Caldwell et al. 1992). And V probably eluded detection until now due to its very low apparent surface brightness. Our And V measurement corresponds to an extinction-free $V$ central surface brightness of $25.2 \mathrm{mag} / \operatorname{arcsec}^{2}$.

\section{Discussion}

The discovery of And V increases the number of M31 dwarf spheroidals from three to four. It changes somewhat the properties of M31's satellite system, as discussed below. A more complete description of M31's entourage of dwarf spheroidals will be presented once our survey and the necessary follow-up observations are complete.

The spatial distribution of the companions to M31 has been discussed by Karachentsev (1996). The discovery of And V changes somewhat the spatial distribution of the M31 satellites. The location of And V relative to M31, M32, M33, NGC 147, 185 \& 205, and And I, II \& III is shown in Figure 2. Curiously, And I, II \& III are all located south of 
M31, while the three more luminous dwarf elliptical companions NGC 147, 185 \& 205 are all positioned north of M31. Also, Karachentsev (1996) notes that there are more M31 companions overall south of M31 than north of M31. And V's location north of M31 lessens both of these asymmetries. Karachentsev (1996) called attention to the elongated shape of the M31 companion spatial distribution (based on the previously known galaxies labelled in Figure 2, plus the more distant galaxies IC 1613, IC 10, and LGS 3, which he argues are also associated with M31) with axis ratio 5:2:1. And V is located within this flattened spatial distribution. With a projected radius from the center of M31 of $112 \mathrm{kpc}$, And V increases slightly the mean projected radius of the M31 dwarf spheroidals from $86 \mathrm{kpc}$ to 93 kpc. Finally, Karachentsev (1996) also discussed morphological segregation among the M31 companions, with the dwarf ellipticals and dwarf spheroidals located closer to M31, and the spirals and irregulars on the outskirts (see also van den Bergh 1994). And V's distance from M31 and our classification of this galaxy as a dwarf spheroidal support the presence of morphological segregation.

The discovery of nearby dwarf galaxies like And $\mathrm{V}$ augments the faint end of the luminosity function of the Local Group. We do not yet have a reliable $M_{V}$ value for And $\mathrm{V}$, but it appears to be similar to that of And III $\left(M_{V}=-10.2\right)$ since they have similar extinction-corrected central surface brightness. From a survey of nine clusters of galaxies, Trentham (1998) derived a composite luminosity function that is steeper at the faint end than that of the Local Group (see his Figure 2). He attributed the difference to poor counting statistics and/or incompleteness among the Local Group sample. The discovery of And V reduces slightly the discrepancy between the Local Group luminosity function and the extrapolation of Trentham's (1998) function. However, many more galaxies would be required to equate the two functions (for example, the difference is $29 \pm 14$ galaxies in Trentham's faintest two bins, $-13 \leq M_{B} \leq-11$ ). 
Based on And V's smooth stellar distribution, lack of $\mathrm{H} \alpha$ and IRAS emission, and absence of young stars in the color-magnitude diagram, we conclude that And $\mathrm{V}$ is a dwarf spheroidal galaxy. A deeper color-magnitude diagram, featuring a bluer color like $B$, and an $\mathrm{H}$ I search would allow us to evaluate more carefully whether And $\mathrm{V}$ could be a transition object between dwarf spheroidals and dwarf irregulars, like LGS 3 but older (see Lee 1995, Mould 1997, Young \& Lo 1997). Based on the available data however, And V is dwarf spheroidal companion to M31, and its stellar population is not obviously different from those of And I or III.

The Digitized Sky Surveys were produced at the Space Telescope Science Institute under U.S. Government grant NAG W-2166. The survey images used here are based on photographic data obtained using the Oschin Schmidt Telescope on Palomar Mountain. The Second Palomar Observatory Sky Survey was made by the California Institute of Technology with funds from the National Science Foundation, the National Geographic Society, the Sloan Foundation, the Samuel Oschin Foundation, and the Eastman Kodak Corporation. The Oschin Schmidt Telescope is operated by the California Institute of Technology and Palomar Observatory. Supplemental funding for sky-survey work at the STScI is provided by the European Southern Observatory. We appreciate the efforts of Barry Lasker and Jesse Doggett at STScI and Watanabe Masaru at the National Astronomical Observatory of Japan in helping us to access the DSS data. We are grateful to Nelson Caldwell, Gary Da Costa, and Jay Gallagher for comments on an earlier version of this manuscript. JED was supported by the NSF Research Experiences for Undergraduates Program at NOAO during the summer of 1997. GHJ wishes to thank Peter Strittmatter for providing a sabbatical office at Steward Observatory during the period when this paper was written. 


\section{REFERENCES}

Aaronson, M., Gordon, G., Mould, J., Olszewski, E., \& Suntzeff, N. 1985, ApJ, 296, L7

Armandroff, T., Boroson, T., De Veny, J., Heathcote, S., Jacoby, G., Lauer, T., Massey, P., Reed, R., Valdes, F., \& Vaughnn, D. 1998, NOAO CCD Mosaic Imager User Manual (Tucson: NOAO)

Armandroff, T. E. 1994, in ESO/OHP Workshop on Dwarf Galaxies, ed. G. Meylan \& P. Prugniel (Garching: ESO), 211

Armandroff, T. E., Da Costa, G. S., Caldwell, N., \& Seitzer, P. 1993, AJ, 106, 986

Bertelli, G., Bressan, A., Chiosi, C., Fagotto, F., \& Nasi, E. 1994, A\&AS, 106, 275

Bremmes, T., Binggeli, B., \& Prugniel, P. 1998, A\&AS, 129, 313

Burstein, D., \& Heiles, C. 1982, AJ, 87, 1165

Caldwell, N., Armandroff, T. E., Seitzer, P., \& Da Costa, G. S. 1992, AJ, 103, 840

Condon, J. J., Cotton, W. D., Greisen, E. W., Yin, Q. F., Perley, R. A., Taylor, G. B., \& Broderick, J. J. 1998, AJ, 115, 1693

Da Costa, G. S. 1998, in Stellar Astrophysics for the Local Group: A First Step to the Universe, ed. A. Aparicio \& A. Herrero (Cambridge: Cambridge University Press), in press

Da Costa, G. S., \& Armandroff, T. E. 1990, AJ, 100, 162

Da Costa, G. S., \& Armandroff, T. E. 1995, AJ, 109, 2533

Da Costa, G. S., Armandroff, T. E., Caldwell, N., \& Seitzer, P. 1996, AJ, 112, 2576

Davies, J. I., Disney, M. J., Phillipps, S., Boyle, B. J., \& Couch, W. J. 1994, MNRAS, 269, 349

Dean, J. F., Warren, P. R., \& Cousins, A. W. J. 1978, MNRAS, 183, 569 
Ellis, G. L., Grayson, E. T., \& Bond, H. E. 1984, PASP, 96, 283

Ferguson, H. C., \& Binggeli, B. 1994, A\&A Rev., 6, 67

Freedman, W. L., \& Madore, B. F. 1990, ApJ, 365, 186

Fusi Pecci, F., Buonanno, R., Cacciari, C., Corsi, C. E., Djorgovski, S. G., Federici, L., Ferraro, F. R., Parmeggiani, G., \& Rich, R. M. 1996, AJ, 112, 1461

Gallagher, J. S., \& Wyse, R. F. G. 1994, PASP, 106, 1225

Jones, J. H. 1993, AJ, 105, 933

Karachentsev, I. 1996, A\&A, 305, 33

Karachentseva, V. E., \& Karachentsev, I. D. 1998, A\&AS, 127, 409

Kennicutt, R. C. 1994, in The Local Group: Comparative and Global Properties, ed. A. Layden, R. C. Smith \& J. Storm (Garching: ESO), 28

Landolt, A. U. 1992, AJ, 104, 340

Lasker, B. M., \& Postman, M. 1993, in ASP Conf. Ser. 43, Sky Surveys: Protostars to Protogalaxies, ed. B. T. Soifer (San Francisco: ASP), 131

Lee, M. G. 1995, AJ, 110, 1129

Lee, M. G., Freedman, W. L., \& Madore, B. F. 1993, ApJ, 417, 553

Massey, P., \& Armandroff, T. E. 1995, AJ, 109, 2470

Massey, P., Armandroff, T., De Veny, J., Claver, C., Harmer, C., Jacoby, G., Schoening, B., \& Silva, D. 1997, Direct Imaging Manual for Kitt Peak (Tucson: NOAO)

Mould, J. 1997, PASP, 109, 125

Mould, J., \& Kristian, J. 1986, ApJ, 305, 591

Mould, J., \& Kristian, J. 1990, ApJ, 354, 438

Nilson, P. 1973, Uppsala General Catalogue of Galaxies, Uppsala Astron. Obs. Ann. 6 
Pritchet, C. J., \& van den Bergh, S. 1988, ApJ, 331, 135

Reid, I. N., Brewer, C., Brucato, R. J., McKinley, W. R., Maury, A., Mendenhall, D., Mould, J. R., Mueller, J., Neugebauer, G., Phinney, J., Sargent, W. L. W., Schombert, J., \& Thicksten, R. 1991, PASP, 103, 661

Reid, N., \& Djorgovski, S. 1993, in ASP Conf. Ser. 43, Sky Surveys: Protostars to Protogalaxies, ed. B. T. Soifer (San Francisco: ASP), 125

Renzini, A., \& Buzzoni, A. 1986, in Spectral Evolution of Galaxies, ed. C. Chiosi \& A. Renzini (Dordrecht: Reidel), 195

Rice, W., Lonsdale, C. J., Soifer, B. T., Neugebauer, G., Kopan, E. L., Lloyd, L. A., de Jong, T., \& Habing, H. J. 1988, ApJS, 68, 91

Sakai, S., Madore, B. F., \& Freedman, W. L. 1997, ApJ, 480, 589

Schmidt, K.-H., \& Boller, T. 1992, Astron. Nachr., 313, 189

Schombert, J. M., Bothun, G. D., Schneider, S. E., \& McGaugh, S. 1992, AJ, 103, 1107

Stetson, P. B. 1987, PASP, 99, 191

Stetson, P. B., Davis, L. E., \& Crabtree, D. R. 1990, in ASP Conf. Ser. 8, CCDs in Astronomy, ed. G. H. Jacoby (San Francisco, ASP), 289

Trentham, N. 1998, MNRAS, 294, 193

van den Bergh, S. 1972a, ApJ, 171, L31

van den Bergh, S. 1972b, ApJ, 178, L99

van den Bergh, S. 1974, ApJ, 191, 271

van den Bergh, S. 1994, ApJ, 428, 617

Weinberger, R. 1995, PASP, 107, 58

Yang, H., \& Skillman, E. D. 1993, AJ, 106, 1448 
Young, L. M., \& Lo, K. Y. 1997, ApJ, 490, 710 
Fig. 1. - The left panels show images of And II \& III from the digitized POSS-II. The right panels show the results of applying our digital enhancement procedure to these POSSII images. The signatures of the galaxies are much more clearly visible in the processed images. The And III processed image also shows a "doughnut" artifact resulting from a very bright, saturated star. Each panel is 17 arcmin on a side. North is at the top, and East is to the left. 


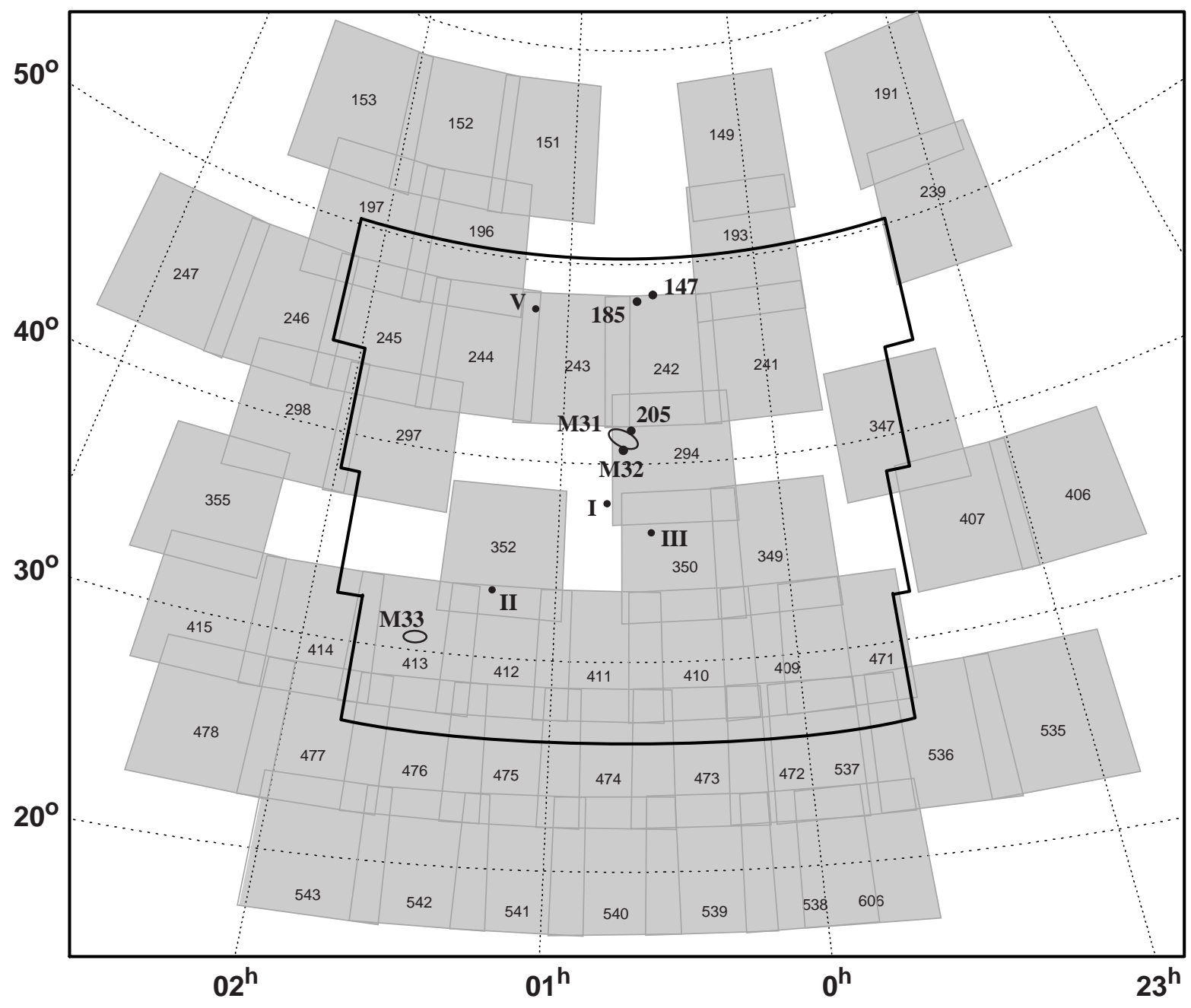

Fig. 2.- Map of the region of sky around M31 that has been surveyed. Individual POSS-II plates that have been searched are shown and labelled. The area surveyed by van den Bergh (1972a, 1974) is thick-outlined. M31 and its known neighbors are labelled. The new M31 dwarf spheroidal, And V, is also indicated.

Fig. 3.- The left panel shows an image of And V from the digitized POSS-II. The right panel shows the effect of applying our digital enhancement procedure to the POSS-II image. Each panel is 17 arcmin on a side. North is at the top, and East is to the left. Note the resemblance between And V and the Figure 1 images of And II \& III. 
Fig. 4.- Image of And V made from the combination of three 900-second exposures through the $V$ filter with the KPNO 4-m telescope. North is at the top, and East is to the right. The image is 7 arcmin (1000 pixels) on a side; And V's center is at $x \approx 500, y \approx 514$.

Fig. 5.- An $\mathrm{H} \alpha$ image of And V made from the combination of five 600-second exposures with the KPNO 4-m telescope is shown in the left panel. A continuum-subtracted version of this image is displayed in the right panel. In each panel, North is at the top, and East is to the right. Each image is 2.7 arcmin on a side. The residuals in the subtracted image around many of the brighter stars are due to small differences in the PSF between $\mathrm{H} \alpha$ and $R$. Note the lack of diffuse $\mathrm{H} \alpha$ emission or $\mathrm{H}$ II regions. 


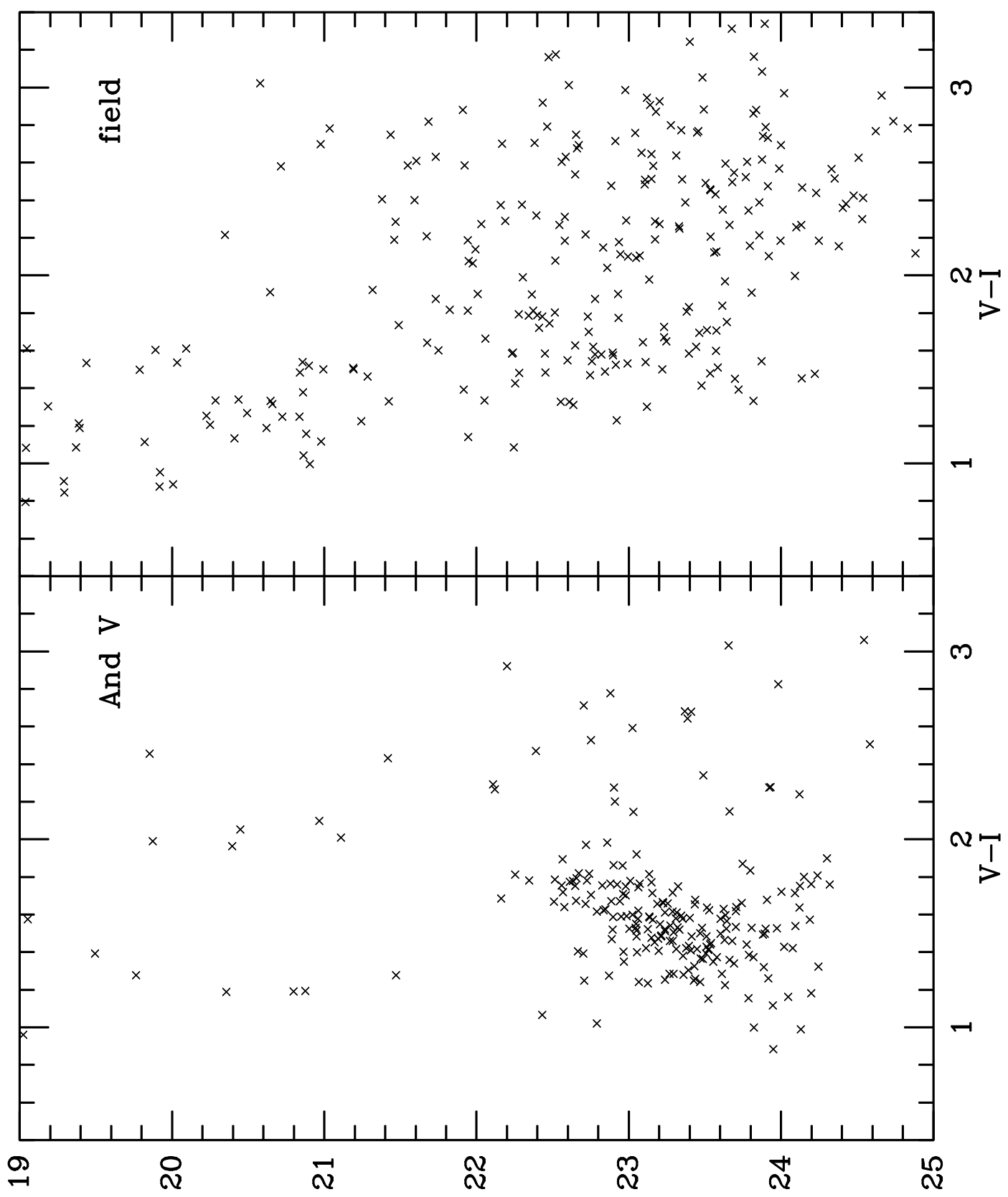

$\Lambda$

Fig. 6.- Color-magnitude diagrams for stars on the And V frames. The left panel shows stars within a radius of 170 pixels (71 arcsec) of the center of And V, where And V members outnumber field contamination. The right panel displays stars greater than 400 pixels (168 arcsec) from the center of And V, where the contribution from And V stars is negligible. The right panel (field) represents 5.5 times more area on the CCD frame than the left panel (And V). Note the upper red giant branch that is present in the And V color-magnitude diagram, which is absent in the field diagram. 


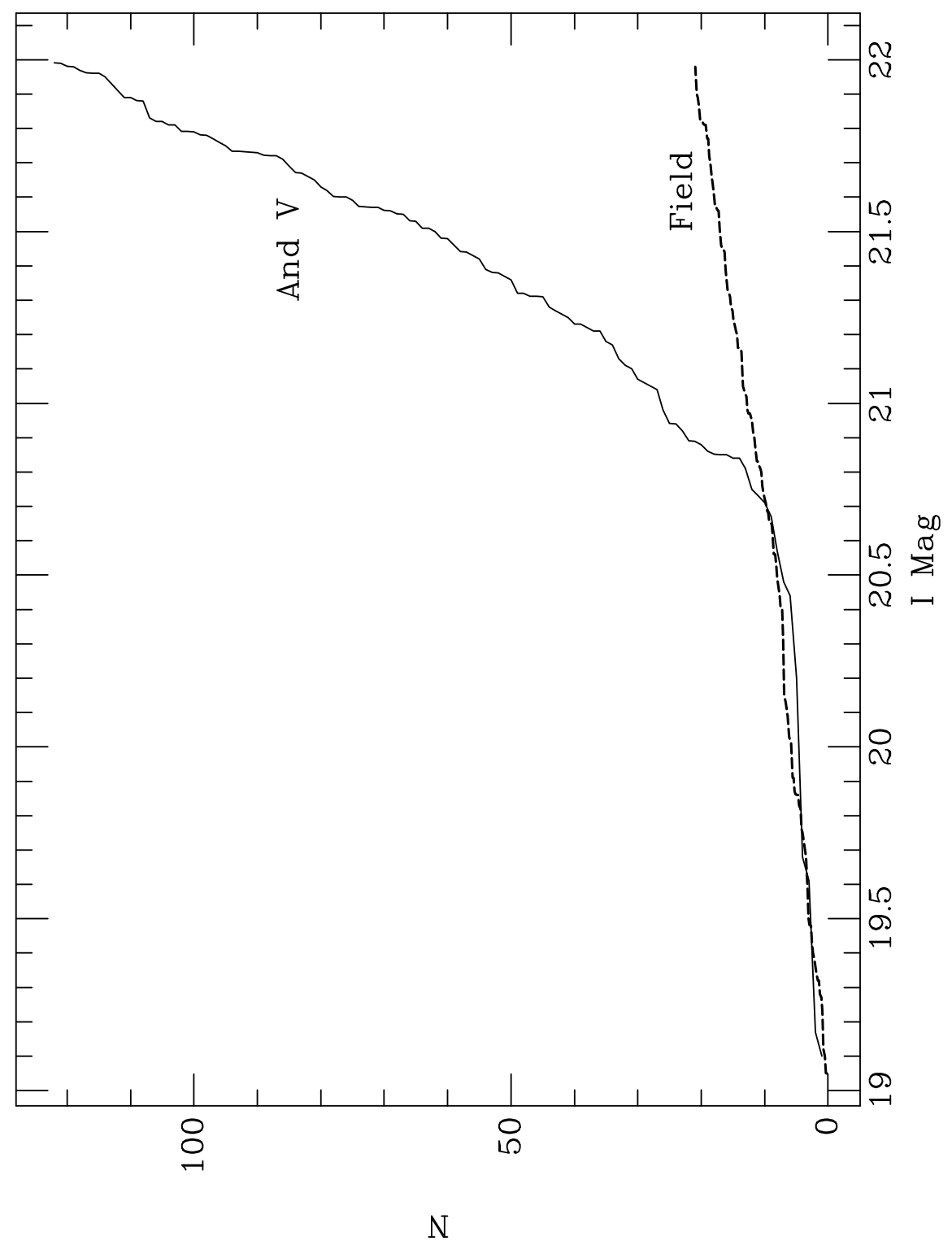

Fig. 7.- Cumulative luminosity functions in $I$ for stars on the And V frames. The solid line shows stars within a radius of 170 pixels $(71 \mathrm{arcsec})$ of the center of And V, where And V members outnumber field contamination. The dashed line represents stars greater than 400 pixels (168 arcsec) from the center of And V, where the contribution from And V stars is negligible. The field luminosity function has been divided by 5.5 because it represents that much larger an area on the CCD frames. Note how the And V luminosity function strongly turns up from the field luminosity function at $I=20.85$, which we interpret as the tip of the And V red giant branch. The use of the cumulative luminosity function (as opposed to the differential luminosity function) avoids binning effects and averages out small random photometric errors. 


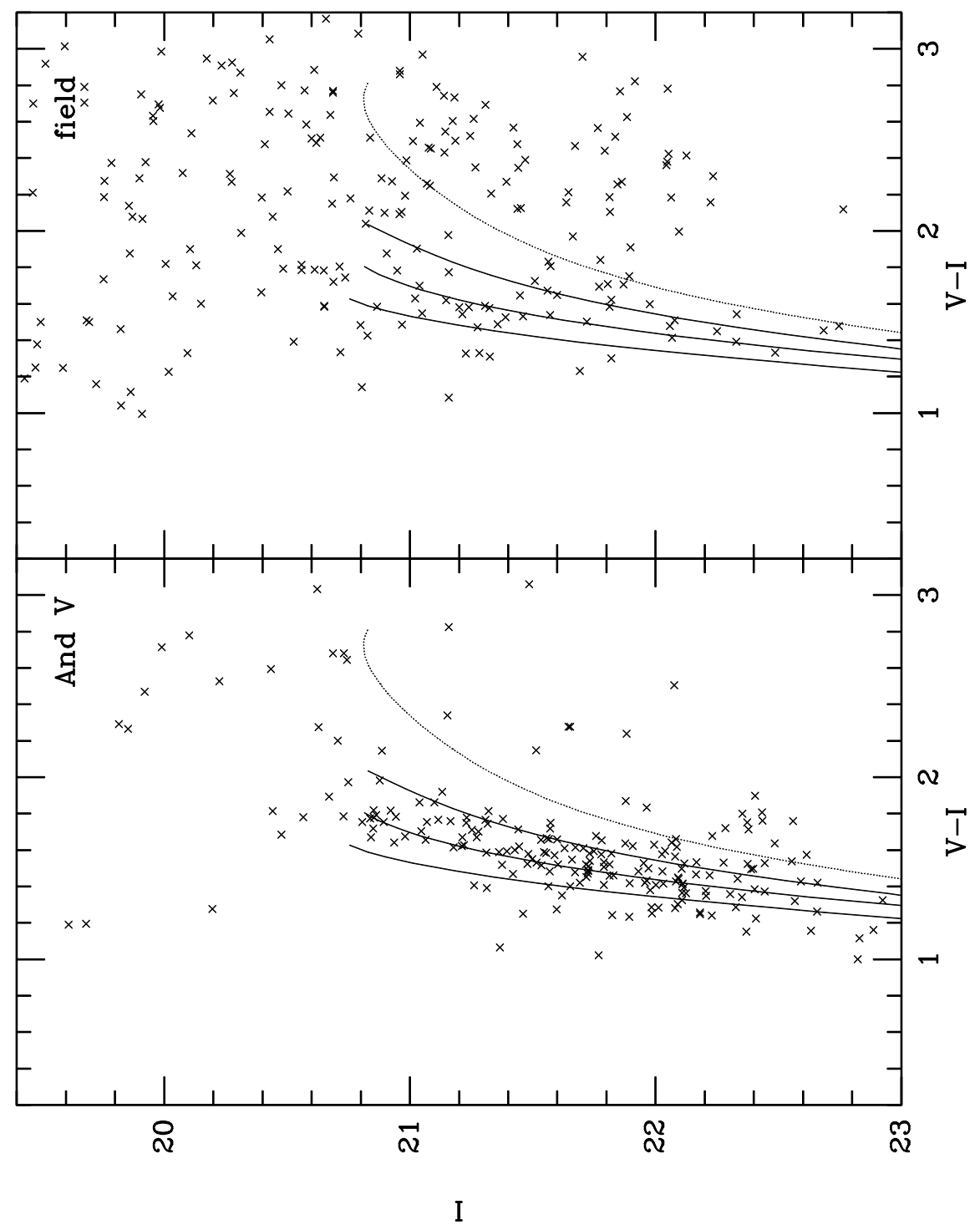

Fig. 8.- Color-magnitude diagrams as in Figure 6, except now with $I$ as the abscissa. Red giant branch fiducials for four Galactic globular clusters that span a range of metal abundance (Da Costa \& Armandroff 1990), shifted to the distance modulus and reddening of And V, have been overplotted. From left to right, the red giant branch fiducials are M15 $([\mathrm{Fe} / \mathrm{H}]=-2.17), \mathrm{M} 2(-1.58), \mathrm{NGC} 1851(-1.16)$, and 47 Tuc $(-0.71$, dotted line $)$. The specifications of the "And V" and "field" regions are given in the text. 
This figure "armandroff.fig1.jpg" is available in "jpg" format from: http://arxiv.org/ps/astro-ph/9807232v1 
This figure "armandroff.fig3.jpg" is available in "jpg" format from: http://arxiv.org/ps/astro-ph/9807232v1 
This figure "armandroff.fig4.jpg" is available in "jpg" format from: http://arxiv.org/ps/astro-ph/9807232v1 
This figure "armandroff.fig5.jpg" is available in "jpg" format from: http://arxiv.org/ps/astro-ph/9807232v1 